\title{
THE FUNERALIZATION PROCESS: TOWARD A MODEL OF SOCIAL TIME*
}

DAVID R. UNRUH

Wichita State University

Mid-American Review of Sociology, 1976, Vol. 1, No. 1:9-25

This paper attempts to describe, categorize, and analyze interactions between social actors and the temporal aspects of a specific social occasion. The burial process, as a social occasion, necessitates the coordination and management of social time between individuals, institutions, and the functionaries of death. In the analysis a temporal "model" is constructed whereby interactions between social actors and the temporal components may be categorized. This temporal "model" consists of: temporal awareness, the temporal epoch, temporal allocations, and aspects of temporal management.

The temporal aspects of situations seem to be important factors in any social pattern. Social interaction requires the coordination of time which tends to limit all forms of social participation (Cottrell, 1940:39). An orientation to time is omnipresent in human interaction. "Interactants attend to time not merely as an abstraction referring to the unending flow of events but, more correctly, to the orderly flow of those events" (Speier, 1973:42). As participants construct activity together they attend to the demands of that activity over the course of time.

Individuals, institutions, and entire cultures may vary widely as to their cognizance of time (Cf. Hall, 1959; Moore, 1963; and Yaker, Osmond, \& Cheek, 1972). Variances in temporal perspectives and restrictions may similarly vary between what Goffman has termed social occasions. Specific examples of such variances in time perspectives, the affective qualities, or attempts to manage time are evident in social parties, concerts, athletic events, and other situations bound by both space and time. ${ }^{1}$

*A slightly different version of this paper was presented at the Midwest Sociological Association meetings in St. Louis, 1976. 
Some social occasions signify a change of status for an individual or society. These rites de passage seem to be particularly bound by the constraints of time. These social rituals serve to signify the transition between the social roles or functions of an individual, or a group of individuals. Speier (1973:43) perceives the importance of time in such 'status transitions:

The fabric of human relationships is understood in part by temporal orientations with which the relationships come into being, are sustained, and are eventually terminated. Birth and death are perhaps the most notably crucial time-bound events which either create new human relationships or cancel old ones.

The funeralization ${ }^{2}$ process is not only a means by which human relationships are cancelled but it is also a vehicle by which bereaved family members may proceed from one stage of their life to another. Not only do audience members come to realize the status transition between life and death for the deceased, but bereaved family members are also reassigned new roles as they continue their own life cycles.

\section{THE DATA}

The data presented were collected through the use of extensive interviews with, and observations of, three diverse funeral establishments in the Wichita, Kansas, area. The sample included a black funeral home, a Catholic and Jewish funeral home, and a Protestant funeral complex. The homes were chosen so as to include a small, medium, and large funeral home, where size was measured by the number of funerals per year. A cross-section of thirty-six funeral services, or about twelve per home, were observed in detail.

\section{THE NOTION OF TEMPORAL AWARENESS}

The funeralization process is composed of a myriad of temporal considerations of which participants must be aware. Funeral home personnel and bereaved family members alike must remain cognizant of the temporal qualities of the situation so that behavior may proceed normally and believably. If some awareness of the temporality of the situation is not shared between interactants, legitimate organization for interaction may not occur.

Temporal awareness, or the realization, perception, and knowledge of the temporal qualities of a situation, is the means by which interaction in a situation is structured and coordinated. In the funeralization process, specific timetables must be composed, activities must be coordinated, negotiations relative to funeral activities must be made, and a diversity of other activities must be mediated simultaneously.

The awareness of time and its obligations seems to be of concern to both funeral home personnel and audience members. Due to the nature of the research, primary attention will be given to manifestations of temporal awareness by funeral home personnel relative to their performances. Analysis of temporal awareness will be categorized using the dramaturgical frontstage/backstage dichotomy. In this context, frontstage will refer to expressions of temporal awareness between funeral home personnel and audience members. Backstage awareness will be that expressed singularly between either funeral home personnel, bereaved family members, or other audience members.

\section{Frontstage Awareness}

It is the funeral director who must guide, manage, and control both the bereaved family and other audience members through the processes of funeralization. Funeral home personnel may attempt to instill or create a degree of awareness in the minds of the bereaved family by continually stressing an appointment time, telephoning to check on the family, and offering to take control of matters if necessary. The funeral director must also convey to bereaved family members that he is bound by temporal considerations as several services may be in process simultaneously. It may be emphasized that the family should decide on a funeral service time so all of the necessary pre-funeral processes may be set into motion. The family must be made aware that a funeral service time one day hence does not allow for the inclusion of newspaper obituaries and the possible 
completion of all preparatory activities. Expressed temporal awarenesses by bereaved family members are often manifested in queries such as "What is the proper time for a funeral service?" "Should it be on the third day after death?" "When can we view the body?" and "When are the visiting hours?"

\section{Backstage Awareness}

The awareness of time may also occur "behind the scenes" where strategies are plotted and withdrawals are made out of performance areas. Backstage behavior may also consist of those activities occurring prior to the funeral service which are out of the view of audience members. Included in this classification would be the preparatory activities surrounding the corpse.

Embalmers have expressed a considerable awareness of time and, in common-sense terms, a knowledge that "haste makes waste." The embalming process is, according to several embalmers, an activity that cannot be done quickly. Time conscious embalmers have expressed this on numerous occasions. When one embalmer stated that he could "embalm a body in forty-five minutes," another embalmer countered, "Well, it would probably look like it, too. Boy, they turn up that embalming machine and they get a little swelling here and there, but they don't care about that." Similarly, one funeral director describes the benefits of taking as much time as needed.

Many guys say they are "hotshot" embalmers and in twenty or thirty minutes they are finished.... The one thing I'm concerned with is "If you don't do your work (properly) in the back room, you can't do your work up front."

Temporal awareness might be seen as an important component in both the front and back stages of the funeral home. It has been demonstrated how funeral home personnel must mediate and control the temporal aspects of the situation so that a myriad of activities may proceed normally and in a totally believable context.

\section{THE TEMPORAL EPOCH}

The entire funeralization process consitutes what will be termed a temporal epoch. A temporal epoch is an extended period of time characterized by a distinctive development or event. $^{3}$ This concept presupposes that the development, or event, is seen by social actors as characteristic of a temporal period. In this instance, the death of an individual and the accompanying rituals of funeralization seem to be the dominant and characteristic events.

The temporal epoch implies a distinguishable point of initiation and cessation of the process. The funeralization process is initiated immediately upon, or shortly after, the death of an individual. Cessation will occur upon the final closing of the grave by cemetery personnel.

Rather than being one continuous process, or several independent processes, the funeralization process is composed of a series of small interrelated components. While the entire process might be initiated upon the death of an individual and culminate with the last shovel of earth on the grave, that does not realistically represent the process. Instead, the process consists of several small temporal periods to be broken down for analysis. These periods may not be clearly and distinctly defined and funeral participants may not be cognizant of the specific points in time in which they pass into a new temporal period. The temporal epoch must be allocated, allotted, and delineated according to the specific functions desired.

\section{THE ÁLLOCATION OF TIME}

The funeralization process per se consists of a number of smaller, interrelated processes which tend to structure activity. Individuals involved in the funeralization process must therefore apportion segments of time out of the larger temporal epoch. The notion of temporal allocation is of primary concern to the funeral director since she/he is the controlling force by which activity is structured and organized for bereaved family members. 
The funeral director, as organizer and supervisor, is in a position where groups of individuals, often experiencing severe emotional distress, are very much dependent on his actions. It is the funeral director who must appear in control of the situation at all times. The funeral director must continually apportion his time between several activities so that the illusion of control is maintained. These activities may be the preparation and arrangements for several funeral services, community activities, or bookkeeping functions within the establishment. Similarly, allocations and segmentations of time relative to the interpersonal transactions between funeral director and bereaved family members must be mediated by the funeral director.

Arrangements for, and conduction of, a funeral service requires the funeral director to negotiate the structure of the funeral service in accordance with desires of family members and establishment capabilities. Conflicts due to other funeral services are often handled through the direct manipulation of family members by the funeral director. Other funeral service times may be suggested, alternative locations may be proposed, or even an entire alteration of the specific day may be maneuvered.

The funeral director must, however, accommodate himself to deviations relative to temporal customs. In most cases, the black funeral service occurs anywhere from four to six days succeeding death whereas the white funeral will generally occur on the third day following death. In the words of a funeral director at a black funeral home,

In our case, most of our services vary from about four to six days. We have a situation in our culture in which we aren' $t$ "instant disposal" types. People... take off weeks from work just to be here. In our culture, births, weddings, and deaths are a cohesive event that brings everybody together.

Funeral establishments specifically catering to the needs of the black community are prepared for such delays whereas a funeral home with a white, Protestant clientele may need to revise their timetables when serving a black client. The delay of several days, usually due to travel time for friends and relatives, may cause the unaccustomed funeral director to reschedule or renegotiate other funeral services. ${ }^{4}$ Similarly, the traditional funeral director may not be accustomed to the extended period of contact with family members that characterizes this extended funerary period.

Temporal allocations are made for both funeral director and bereaved family members. Allocations of time have been delineated for specific processes in the overall funeralization process. Processes delineated for funeral home personnel are: the first call, body pick-up, the embalming process, the arrangement process, covert arranging and negotiation, the actual funeral service, and closing the grave. Conversely, temporal allocations for the bereaved are much less formalized. Delineations consist of: notification of death, waiting periods, contact with funeral directors, the arrangement process, the visitation period in which the deceased is viewed by family members, the funeral service, and the final journey home (see figure 1.).

It seems fairly obvious that the preceding temporal allocations are indistinct and arbitrarily delineated. In a similar manner, it is an obvious fact that temporal allocations seem to be most constricting to funeral home personnel. This is due, in part, to the specific function the funeral director plays in the society. It is precisely his job to assume the role of funeral director. Therefore, he is expected to take over and control the situation while family members have time to adjust to their loss.

It is in this role of funeral director that funeral home personnel control particular aspects of temporal allocations. Control, or manipulation, of the temporal aspects of a funeral service may be necessary because of known idiosyncrasies of particular religious denominations or individual ministers. For instance, funeral homes often maintain a part-time chaplain to be used by families who do not have a family minister. They are then free to call upon this individual when, and if, the families should so desire. Activity may be structured by this very activity. Since the minister does not know the family or the deceased, chances are that he won't be able to speak at great length about the better qualities of the deceased. The black funeral home referred to their chaplain as seven-minute Smith since he could say 


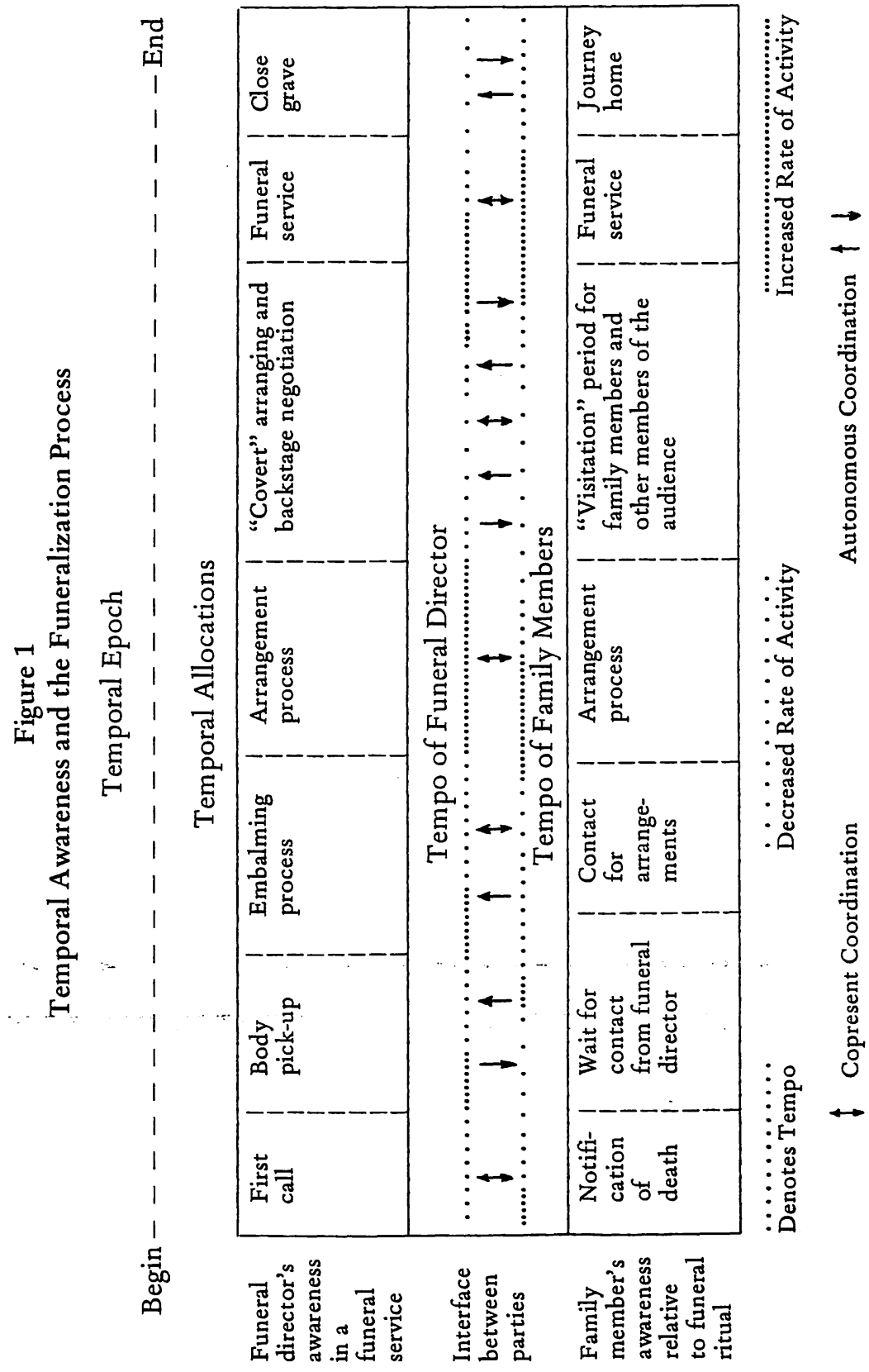

what he had to say in seven minutes and get out of there. On the other hand, specific ministers may cause funeral directors to cancel all other activities for the day. A Baptist minister, very well known to the funeral home personnel, not unexpectedly conducted a funeral service that lasted well over two and one-half hours. The funeral home personnel knew full well that no other activity could be planned for the entire afternoon when a funeral service was conducted by this minister.

\section{ASPECTS OF TEMPORAL MANAGEMENT}

The temporal aspects of situations are influenced, synchronized, and coordinated through the management of components of interaction within the temporal order. Primary components of this temporal sphere which are manageable, both individually and collectively, might be the tempo and timing aspects of the temporal order.

Tempo may best be defined as the number of events, both social and psychological, that occur in a given unit of time. Timing seems to consist of the coordination or regulation of separate pulsations, activities, or processes. ${ }^{5}$ These concepts must necessarily be viewed as interrelated and interacting elements.

Activity within the funeral process fluctuates with the current demands of the situation. As a result, funeral home personnel, bereaved family members, and other members of the audience are continually adjusting and re-adjusting to situational demands on temporal usage. Thus, the funeralization process consists of a series of temporal ups and downs that require adjustment and mediation by social actors.

\section{Pre-Funeral Activities}

Notifications of death are received by funeral establishments at all hours of the day and night. Depending upon the physical design of the establishment and that establishment's ability to spare one of their personnel during a funeral service, death calls received during a funeral service may be acted upon immediately or they may be postponed until after the funeral service. Should a decision be made to accept the corpse during a funeral service, the 
tempo and timing aspects of the situation become increasingly important. The funeral director must then time his activities at the hospital with those occurring at the funeral home chapel. A multiplicity of events must here be coordinated simultaneously.

The converse of such timed and coordinated behavior is evident at the funeral home when a death call is received and no other activities are planned for the day. The excursion to the hospital, or other place of death, may then be used to accomplish personal errands as well as other activities for the funeral establishment. These extraneous factors do not significantly affect the embalmer's performance since prompt action is not a critical component of the situation and precise timing is not necessary.

The phenomenon of the death call may also require a degree of coordinated behavior between funeral director, hospital personnel, and bereaved family members. Several of the funeral establishments studied purposefully delayed action toward acquiring the corpse until they were positive the families had been removed from the place of death. In other words, not only is precise timing not important here but the converse may be true. The funeral director may wait up to several hours before receiving the corpse becomes convenient.

Once back at the funeral establishment, the coordination and tempo at which activity proceeds is similarly influenced by a temporal framework.

Throughout the course of activities prior to the funeral service itself, the funeral director must attempt to obtain the proper name, place of death, and other information relative to the deceased. This information is generally elicited from bereaved family members directly, but situations do arise where such information is obtained from hospital personnel and involved others. This process of eliciting the vital statistics may have to be accomplished in a short period of time. Although the activity may have to occur quickly, it should not proceed too fast so as to foster the impression of carelessness, hastiness, and even eagerness on the part of the funeral director. Although the temporal period may be brief, the funeral director must appear as though he has all the time in the world. Should he fail in this aspect of impression management and appear somewhat hurried and impatient, the funeral director risks destroying the bereaved family's image of him as one who serves patiently and whenever needed. Therefore, the funeral director must proceed as usual even though his timetable for the day might be significantly altered or destroyed.

The tempo at which the funeralization process moves is somewhat predetermined by the prescribed period between death and the scheduled funeral service. It is a general rule that the funeral service occur on the third day after death. This three-day period seems to be preferred by both funeral home personnel and bereaved family members for several reasons. According to one funeral director,

the family responding to the loss needs some time and so it's important that the funeral service time be set up to give the people time to respond to the loss.... The most common response is that "I'm just in a fog, it hit me like a ton of bricks." The funeral director does not want to go in and press them to make a response immediately without giving them some time to compose themselves.

Coordinations similarly occur relative to conflicting functions or activities at the funeral establishment. The funeral director must coordinate and thereby time the processes of one funeral service with others occurring in close temporal proximity. If necessary, funeral services are coordinated in such a way that a graveside service, a church service, and a funeral home chapel service could all be scheduled within an hour or two of.each other. Such schedulings require the coordination of facilities, automobiles, and funeral home personnel. A similar coordination and negotiation must occur between funeral home personnel so that audience members are guided and manipulated into the proper performance area.

Preparation for the funeral service itself illustrates the fluctuating nature of the rate and coordination of activity. The tempo of activity depends greatly on the unique situational variables that place each funeral service on a novel space/time coordinate. Such variables would include concurrent activities in 
the establishment, weather conditions, relative ease of travel for family members, and the psychological state in which family members enter into the negotiation process.

The period immediately succeeding a death marks a period for the bereaved in which a multiplicity of events occurs simultaneously. Friends and relatives must be notified, a minister may have to be contacted, and the funeralization process must be initiated. This flurry of activity is not to be experienced by bereaved family members to the same degree throughout the entirety of the funeralization process. Increases in tempo do occur during the arrangement process and during the actual funeral service, but time, for the bereaved, may be characterized as proceeding at a relatively slow pace.

Funeral service negotiations and preparations are made in the presence of the bereaved family whenever possible. In this way, if there are any problems, the family is right there to help (them) straighten them out. Thus, by making telephone calls and funeral arrangements with the family present, the funeral director may create the illusion of patient concern while spending the least possible time in the actual arrangement of a funeral service.

\section{Funeral Activities}

Rates of activity just prior to a funeral service might be best characterized as fluctuating. Preparations for a funeral service at 2:00 p.m. actually begin much earlier than that time. The funeral homes under study initiate preparatory activities for a specific service quite early on the day of a funeral service.

Audience members seem cognizant of the fact that the funeral service does -not begin until members of the bereaved family have assumed their positions of prominence in the chapel or sanctuary. It would not be proper for family members to enter the chapel some thirty minutes or so before the funeral service to greet friends.

Precautions against early entrances by the bereaved are taken by all funeral establishments studied. Larger funeral establishments, due to an abundance of space, are capable of shielding family members in a lounge area until the scheduled funeral service time. Funeral establishments without spacious facilities often attempt to arrive by motorcade at precisely the time of service, or just prior to that time. In this aspect of timing, tempo seems to be a function of the timing process. The rate at which the motorcade travels, and the rate at which family members are ushered into the chapel, are easily manipulated to conform to the timetable desired.

Coordinated behavior occurs between funeral home personnel and others through the use of spatial placement. Ministers, organists, and soloists must coordinate their activity with that desired by the family members. Such coordinations occur through the use of both verbal and nonverbal cues. The organist at every funeral home studied allowed herself some means by which she could monitor chapel activity. In most cases, this consisted of a small window, partition, or screen behind which she could unobtrusively monitor activity in the frontstage area.

In many instances, when funeral directors have worked together during numerous funeral services, interaction between them takes on a spontaneous action-reaction quality. The social action of experienced teams of funeral directors is structured and timed according to their previous experiences. In many instances, behavior may be so finely coordinated that a funeral director caught out of position may have his void unconsciously filled by a colleague.

Funeral home personnel and their awareness of the importance of temporal coordination is evidenced by the emphasis on clock time. Clocks are strategically located throughout the funeral establishments and their presence does not go unnoticed. It is not an unusual occurrence for funeral directors to continually glance at, and synchronize their watches throughout the funeral service. Proper coordination of behavior in the funeral service seems to be dependent upon the use of a mechanical means by which interaction may be guided. ${ }^{6}$

Coordinated behavior necessarily occurs between funeral home personnel and bereaved family members during periods of emotional distress and emotional breakdown by the bereaved. The funeral director must decide whether or not to intervene in the consolation process during this period. Once again, past experiences seem to be the foundation upon which the funeral 
director can base his actions. On this basis, the funeral director may wait until the time is right for intervention. In many instances, this intervention is left to members of the family surrounding the bereaved who are felt to be better equipped to deal with the emotional needs of those individuals.

\section{Post Funeral Activity}

The final funeral act, that of cemetery interment, constitutes the final scene in the funeralization process. The solemnity of the occasion is signified by the relatively slow pace at which the procession moves from the scene of the funeral service to the place of interment. Aspects of timing and tempo in this process do not appear, on the face of it, to play the influential role that they do during the rigidly scheduled funeral service. Once the interment ritual is completed, the funeral directors seem to be in no real hurry to vacate the scene. Instead, the funeral directors generally allow friends and family members time to visit and engage in conversation for some time afterward. It is only when this period is deemed excessive that funeral home personnel will attempt to remove the family from the area.

The closing of the grave, which is accomplished only after all audience members have vacated, constitutes an activity accomplished in solitude yet timed and coordinated with precision. Cemetery workers, while waiting for interment services to conclude, are often found hiding behind trees and buildings just out of the sight of audience members. The closing process is timed by these cemetery functionaries to begin immediately as the last automobile in the procession recedes out of the cemetery. Coordination of activity is of particular importance here since cemetery personnel must not only coordinate their behavior with the cessation of the interment service, but in many instances, with activities occurring in another cemetery across the city. This is due to the fact that several cemeteries in the city are managed and operated by one firm with a single set of employees.

\section{DISCUSSION}

A model of temporal awareness may be constructed out of the data presented in the preceding sections. This model, as represented in figure 1 , is a graphic representation of temporal awareness and the funeralization process. It is shown that temporal awareness is a necessary requisite for any of the succeeding concepts. Increasing in specificity is the temporal epoch which merely delineates the beginning and end of a process. Analysis is facilitated by isolating a process out of the broader epoch. Temporal allocation further delineates the process. Units of analysis are created in which a specific activity seems to predominate. Time is allocated according to specific needs and functions to be performed. Figure 1 demonstrates the fact that allocations do not necessarily need to be congruent between parties. The number of processes and activities for funeral home personnel far outnumber those of the bereaved family.

The micro-order of temporal awareness, temporal management, seems to include components of both tempo and timing. It has been demonstrated how the utilization of various tempos may be a way by which timing and coordinated behavior might be achieved. The second component of this temporal micro-order is that of the timing itself. Timing is a way by which individual processes and activities may be coordinated. Timing seems to have both a copresent and an autonomous component. ${ }^{7}$ Copresent timing implies the coordination of behavior between two or more individuals while autonomous timing seems to be of a more mechanical nature.

This model of temporal awareness and the management of time is specifically adapted to only one form of social ritual or occasion. If this model is in any way an accurate and meaningful portrayal of social time, the model should then be applicable to other social rituals, processes, and situations. The specific emphasis of this research is, in a very real sense, exploratory. The temporal aspects of situations have most generally been relegated to a subordinate role in sociological understanding. It is hoped that questions raised by this specific research will serve to stimulate further exploration and inquiry in this direction. 
Mid-American Review of Sociology

\section{NOTES}

1. The further interdependence between space and time in this specific social ritual is demonstrated in my master's thesis. See Death As Drama: An Analysis of The Spatial And Temporal Aspects of Funerary Behavior (unpublished master's thesis, Wichita State University).

2. This paper does not deal with cremation, which is subject to somewhat different temporal processes and procedures.

3. The temporal epoch differs from Lyman and Scott's concept of time track in that the temporal epoch connotes an extended period of time signifying a significant social occurrence rather than a mere transition. See Stanford M. Lyman and Marvin B. Scott, A Sociology of The Absurd. New York: Appleton-Century-Crofts. 1970.

4. Much of the interview data seemed to suggest that many black families would break tradition and engage the services of one of the larger white funeral homes in the city. This break does not last long according to one funeral director because these white establishments are not sensitive to specific needs and the people tend to feel that they were "not treated well."

5. The definitions of tempo and timing are derived from Amos Hawley's human ecology framework. See A.H. Hawley, Human Ecology: $A$ Theory of Community Structures, New York: Ronald Press, 1950 Also applied in W.R. Rosengren and S. DeVault's "The Sociology of Time and Space in an Obstetrical Hospital" in E. Friedson (ed.) The Hospital in Modern Society, The Free Press of Glenco, 1963.

6. Clock time, as used here, is very much related to the concept of mechanical timirg as presented in the work of Carl Cuuch et al. See Couch, Leichty, Anderson, and Hintz, "Timing and Social Life" in Constructing Social Life: Keadings in Behavioral Sociology From The Iowa School. Champaign, Ill.: Stipes Publishing Co., pp. 124-30.

7. Copresent timing is based on the work of Carl Couch et al., "Timing and Social Life" in Constructing Social Life: Readings In Behavioral Sociology From The Iowa School. Champaign, Ill.: Stipes Publishing Co., pp. 124-26.
The Funeralization Process

\section{REFERENCES}

Cottrell, William F.

1940 The Railroader (Stanford, Calif.: Stanford University Press).

Couch, Carl et al.

1975 Constructing Social Life: Readings in Behavioral Sociology From The Iowa School (Champaign, Ill.: Stipes Publishing Co.).

Hall, Edwart T.

1959 The Silent Language (Garden City, New York: Doubleday).

Hawley, Amos H.

1950 Human Ecology: A Theory of Community Structure (New York: Ronald Press Inc.).

Lyman, Stanford M. and Marvin B. Scott

1970 A Sociology of the Absurd (New York: Appleton-Century-Crofts).

Moore, Wilbert E.

1963 Man, Time, and Society (New York: John Wiley and Sons Inc.)

Rosengren, William R. and Spencer DeVault

1963 "The Sociology of Time and Space in an Obstetrical Hospital" in The Hospital in Modern Society, Elliot Friedson, ed. (New York: The Free Press).

Speier, Matthew

1973 How to Observe Face-To-Face Communication: 'A Sociological Introduction (Pacific Palisades, California: Goodyear Publishing Co. Inc.).

Unruh, David R.

1976 "Death As Drama: Analysis of the Spatial and Temporal Aspects of Funerary Behavior" (unpublished master's thesis, Wichita State University).

Yaker, Henri et al.

1972 The Future of Time: Man's Temporal Environment (Garden City, New York: Doubleday). 